\title{
Dos nuevas especies del género Volvarina Hinds, 1844 (Mollusca: Neogastropoda: Marginellidae) de Venezuela
}

\author{
Two new species of the genus Volvarina Hinds, 1844 (Mollusca: Neogastropoda: \\ Marginellidae) from Venezuela \\ Manuel Caballer ${ }^{1}$, José Espinosa ${ }^{2}$ y Jesús Ortea ${ }^{3}$ \\ ${ }^{1}$ Departamento de Oceanología y Ciencias Costeras. IVIC. Ctra. Panamericana Km 11, Miranda, Venezuela \\ ${ }^{2}$ Instituto de Oceanología, Avda. $1^{a} n^{o}$ 18406, E. 184 y 186, Playa, La Habana, Cuba \\ ${ }^{3}$ Departamento BOS, Universidad de Oviedo, España \\ macaball@ivic.ve
}

\begin{abstract}
Two species of the genus Volvarina Hinds, 1844 were collected alive in La Tortuga Island (Venezuela) during the year 2008. None of them fit in the original description of the species of the genus known to date, so they are described as new in this work. Volvarina ivic, new species is characterized by four columellar folds, aligned in pairs, and by the shape and the color pattern of the shell, with three spiral dark pink bands, the latest of which is very wide and bifurcated and also by the pattern of white spots observed in the foot of the living animal. Volvarina latortuga, new species, its characterized by the shape and the small size of its pale shell that has three undivided dark yellow spiral bands and four parallel columellar folds.
\end{abstract}

Key words: Endemisms, South Caribbean, Cariaco, La Tortuga Island

\section{Introducción}

El inventario de las especies antillanas y caribeñas de marginélidos del género Volvarina Hinds, 1844 se ha incrementado en los últimos años, gracias a los aportes de Díaz et al. (1996), Espinosa \& Ortea (1997, 1998, 1999, 2000, 2002, 2003, 2005), Ortea \& Espinosa (1998), Boyer (2001, 2004), Gracia \& Boyer (2004), Espinosa et al. (2007) entre otros; un buen número de estas especies ha sido recopilado por Boyer (2006) e ilustrado por Cossignani (2006).

En el caso particular de Venezuela, las especies conocidas del género hasta el momento son ocho; Volvarina avena (Kiener, 1834) registrada en la Blanquilla (Talavera \& Princz 1985 fide Rosenberg 2009), Mochima (Sucre) (Ramos \& Robaina 1994), la isla de Margarita, Cubagua, Sucre (Macsotay \& Campos 2001), Chacopata (Sucre) (Narciso et al. 2002), Las Rochas (Cossignani 2006), isla de Aves, archipiélago de Los Roques y en isla Orchila (Rosenberg 2009), Volvarina albolineata (d'Orbigny, 1842) en el
Resumen.- Dos especies del género Volvarina Hinds, 1844 fueron recolectadas vivas en isla La Tortuga (Venezuela) durante el año 2008. Ninguna de ellas corresponde con la descripción de las especies conocidas hasta la fecha, por ello se describen como nuevas para la ciencia en este trabajo. Volvarina ivic, especie nueva, se caracteriza por tener los cuatro pliegues columelares alineados por pares y por la forma y el patrón de color de la concha con tres bandas espirales rosa pardo, la última de las cuales es muy ancha y se bifurca, además del patrón de manchas blancas en el pie del animal vivo. Volvarina latortuga, especie nueva, se caracteriza por la palidez, la forma y el pequeño tamaño de su concha con tres bandas espirales pardo amarillentas indivisas y por los cuatro pliegues columelares paralelos entre sí.

Palabras clave: Endemismo, Caribe Sur, Cariaco, Isla La Tortuga

archipiélago de los Roques (Talavera \& Princz 1985 fide Rosenberg 2009) y Chacopata (Sucre) (Narciso et al. 2002), Volvarina taeniata (Sowerby, 1846) en la isla de Margarita (Talavera \& Princz 1985 fide Rosenberg 2009), Volvarina gracilis (CB Adams, 1851) en Sucre (Talavera \& Princz 1985 fide Rosenberg 2009), Volvarina mexicana (Jousseaume, 1875) en el Golfo de Venezuela (Lipe 1991 fide Rosenberg 2009), Volvarina vokesi De Jong \& Coomans, 1988 en Falcon (Wakefield et al. 2002), Volvarina sp. 4 en la isla de Coche (Cossignani 2006) y Volvarina lactea (Kiener, 1841) en la isla de Margarita (Macsotay \& Campos 2001) y en la Quebrada Mar Abajo (Weisbord 1962; como Volvarina lustra Weisbord, 1962). Esta última probablemente sea una especie del género Hyalina Schumacher, 1817. Princz (1978) cita algunos ejemplares en las localidades de Robledal, el Saco y La Restinga de la isla de Margarita, bajo el nombre Marginella avena Kiener, 1834, cuyo estatus taxonómico precisa ser revisado.

Como resultado de los muestreos realizados en isla La Tortuga se han recolectado dos especímenes 
pertenecientes a dos especies no descritas y diferentes entre sí, que se dan a conocer en el presente trabajo.

\section{Material y métodos}

Los ejemplares de ambas especies fueron capturados manualmente mediante buceo en apnea en isla La Tortuga a lo largo del año 2008. Cada uno de ellos fue estudiado en vivo con un microscopio estereoscópico Carl Zeiss, tomando datos sobre su anatomía externa y coloración. Posteriormente los animales fueron fotografiados y conservados en etanol al 96\%. Como complemento se realizaron diagramas de las conchas usando un microscopio estereoscópico Olympus SZ16.

\section{Resultados y discusión}

Familia Marginellidae Fleming, 1828

Género Volvarina Hinds, 1844

Volvarina ivic especie nueva (Figs. 1 A-C y 2 A-B)

Material examinado

Un ejemplar recolectado vivo (30/11/2008) en cayo La

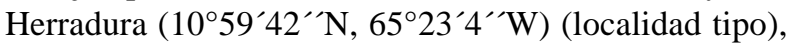
isla La Tortuga, Venezuela, a $1 \mathrm{~m}$ de profundidad, en un fondo de cascajo, designado como Holotipo (9,6 mm de largo y 4,25 mm de ancho). Depositado en las colecciones marinas del Departamento de Oceanología y Ciencias Costeras (DOCC) del Instituto Venezolano de Investigaciones Científicas (IVIC) (número de tipo IVICCMT001).

\section{Descripción}

Concha lisa y brillante, de tamaño mediano, de forma subcilíndrica, con la espira saliente, relativamente corta y aguda. El lado izquierdo de la concha (vista ventral) es convexo, mientras que el derecho es menos convexo y sinuoso hacia su parte media-baja anterior. Protoconcha formada por al menos una vuelta grande, redondeada y saliente, con un núcleo relativamente pequeño. Teleoconcha de tres vueltas, la última ocupa aproximadamente el 88,8\% del largo total de la concha. Abertura estrecha en su porción posterior y marcadamente más ancha en la anterior; labio externo varicoso, relativamente ancho y poco engrosado, insertado en la espira justo por debajo de la sutura de la vuelta precedente. Columela con cuatro pliegues, los dos anteriores más desarrollados, principalmente el segundo que es el mayor de todos; los dos pliegues posteriores están alineados entre sí, al igual que los dos anteriores. Color rosa pálido, casi translúcido en algunas partes, con tres bandas espirales rosa pardo más oscuras, una subsutural, una media y la otra anterior, la cual es muy ancha y se bifurca al final; el labio externo y los pliegues columelares, así como el extremo anterior de la concha, son blancos. La espira y la protoconcha son de color claro, casi blancas.

El cuerpo del animal es hialino algo translúcido, con dos grandes manchas blanco nieve en las zonas anterior y media de los laterales del pie y otras cuatro hacia su extremo posterior. El borde anterior del pie presenta a cada lado unos pequeños palpos superpuestos de un blanco más opaco que el resto del pie. En los tentáculos hay manchitas circulares blanco nieve alternando con manchitas alargadas de color naranja y en el sifón manchones irregulares blanco nieve y algunos puntos naranjas dispersos que también se observan en la parte anterior y posterior del pie. Por la transparencia de la concha, se ven en el manto manchitas anaranjadas, de distribución y forma irregulares.

\section{Etimología}

Volvarina ivic, acrónimo del Instituto Venezolano de Investigaciones Científicas, en el año de su 50 aniversario (2009), como reconocimiento a sus aportes para el desarrollo y avance de la ciencia.

\section{Observaciones}

Por el tamaño, la forma general de la concha y su patrón de color, la disposición de los pliegues columelares alineados por pares y el patrón de color del animal vivo, $V$. ivic, especie nueva, no guarda relación con ninguna otra especie antillana del género con la que pudiera ser comparada.

Cossignani (2006) ilustra como Volvarina sp.4 un ejemplar (6 mm de largo) de forma similar procedente de la cercana isla de Coche $(-30 \mathrm{~m})$, Venezuela, que podría ser un segundo ejemplar de $V$. ivic especie nueva, aunque el patrón de color de la concha seca es blanco uniforme.

Volvarina latortuga especie nueva (Figs. 1 D-F y 2 C-D)

Material examinado

Un ejemplar recolectado vivo (25/09/2008) frente al

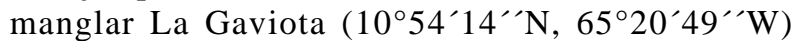
(localidad tipo), costa sur de la isla La Tortuga, Venezuela, a $0,5 \mathrm{~m}$ de profundidad, en un fondo de cascajo, designado como Holotipo (6,5 mm de largo y 2,9 mm de ancho). Depositado en las colecciones marinas del DOCC del IVIC (número de tipo IVICCMT002).

\section{Descripción}

Concha lisa y brillante, de tamaño pequeño comparada con otras especies antillanas del género, de forma subcilíndrica, con la espira muy poco saliente, 

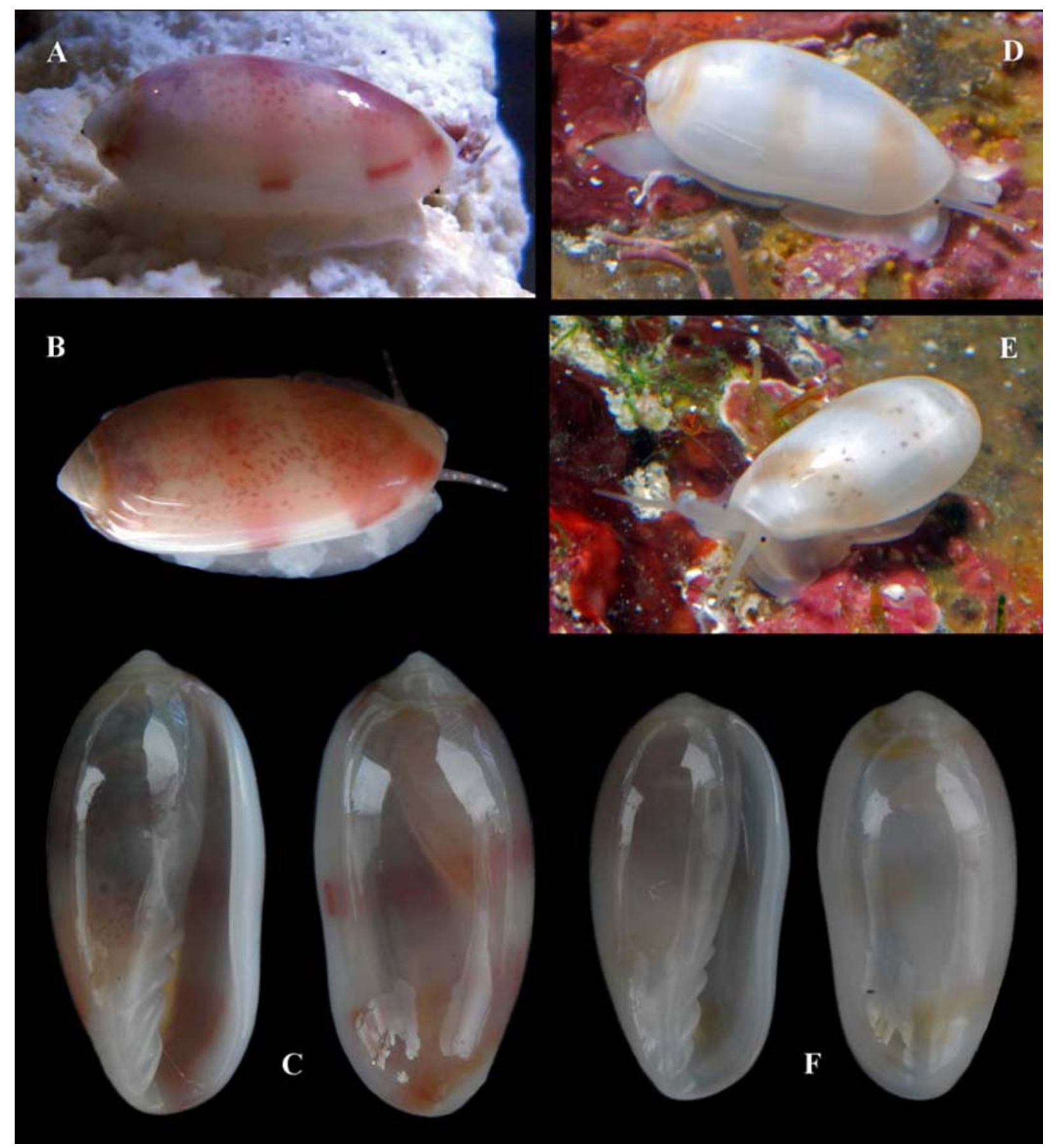

Figura 1

Holotipo de $V$. ivic $\mathrm{n}$. sp. (9,6 mm x 4,25 mm) (A-C), A. animal vivo en vista lateral, B. animal vivo en vista dorsal, C. vistas dorsal y ventral de la concha. Holotipo de V. latortuga n. sp. (6,5 mm x 2,9 mm) (D-F), D. animal vivo en vista lateral, E. animal vivo en vista frontal, F. vistas dorsal y ventral de la concha

Holotype of V. ivic n. sp. (9.6 mm x $4.25 \mathrm{~mm}$ ) (A-C), A. living animal in lateral view, B. living animal in dorsal view, C. ventral and dorsal view of the shell. Holotype of V. latortuga n. sp. (6.5 mm x $2.9 \mathrm{~mm})$ (D-F), D. living animal in lateral view,

E. living animal in frontal view, F. ventral and dorsal view of the shell 

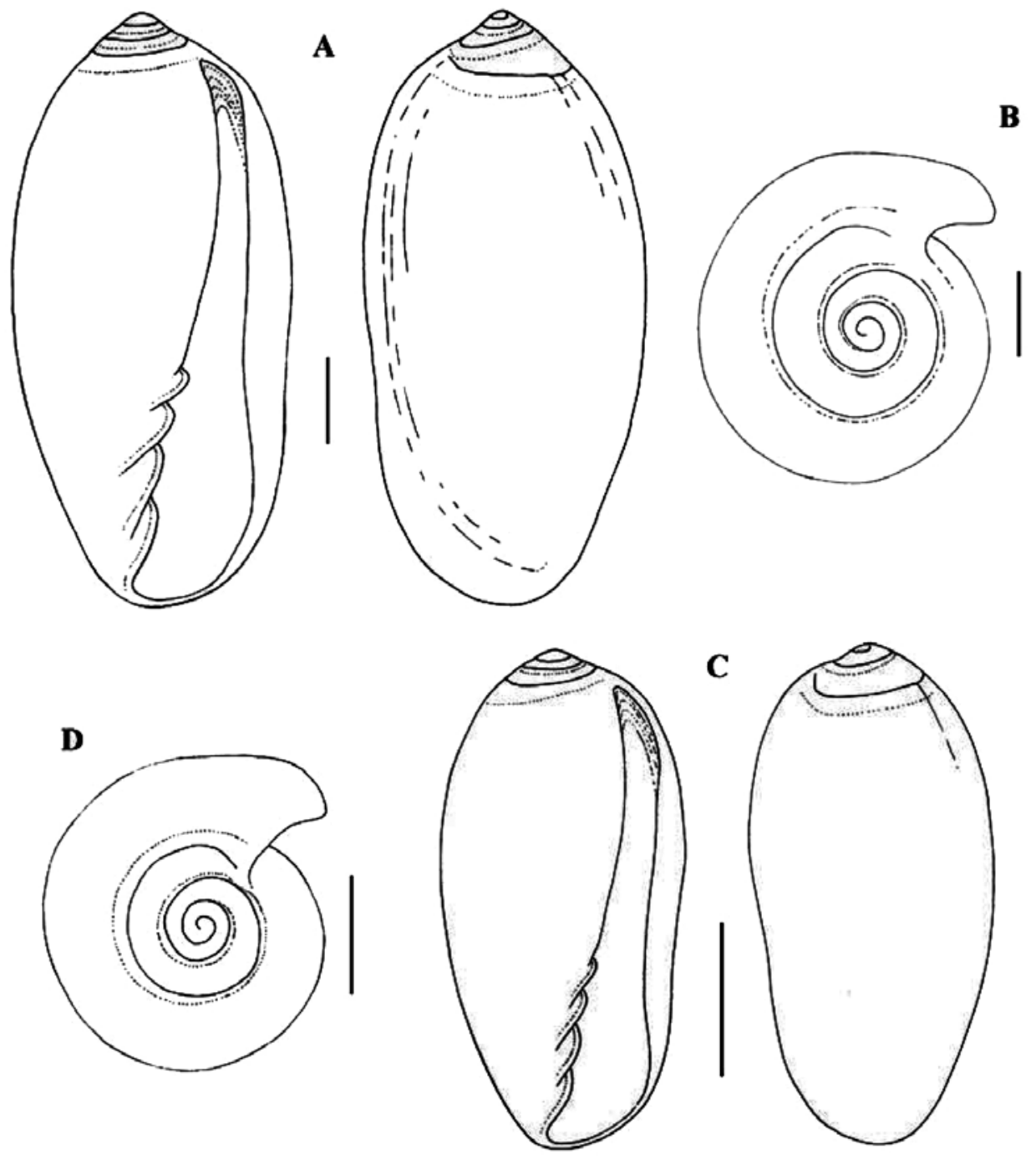

Figura 2

Diagrama de V. ivic n. sp. mostrando la concha en vista ventral y dorsal (A) (escala $2 \mathrm{~mm}$ ) y su protoconcha (B), (escala $1 \mathrm{~mm}$ ). Diagrama de V. latortuga n. sp. mostrando la concha en vista ventral y dorsal (C) (escala $2 \mathrm{~mm}$ ) y su protoconcha (D) (escala $1 \mathrm{~mm}$ )

Line diagram showing the shell of V. ivic n. sp. in ventral and dorsal view (A) (scale bar $2 \mathrm{~mm}$ ) and protoconch (B), (scale bar $1 \mathrm{~mm}$ ). Line diagram showing the shell of $V$. latortuga $\mathrm{n}$. sp. in ventral and dorsal view (C) (scale bar $2 \mathrm{~mm}$ ) and protoconch (D) (scale bar $1 \mathrm{~mm}$ ) 
relativamente corta y algo redondeada. El lado izquierdo de la concha (vista ventral) es convexo, mientras que el derecho es menos convexo y sinuoso hacia su parte media-baja anterior. Protoconcha formada por al menos una vuelta grande, redondeada y moderadamente saliente. La teleoconcha consta de unas dos vueltas, la última de las cuales ocupa aproximadamente el 90,4\% del largo total de la concha. Abertura casi tan larga como el largo total de la concha, estrecha en su porción posterior y algo más ancha en la anterior; labio externo varicoso, relativamente ancho y un poco engrosado, insertado en la espira justo sobre la sutura de la vuelta precedente. Columela con cuatro pliegues casi paralelos entre sí. Color blanco, casi uniforme y translúcido, con tres pálidas bandas espirales de color pardo amarillento, una subsutural, una media y la otra anterior, todas de la misma anchura.

El animal es de color blanco translúcido. Los tentáculos y el sifón son del color del cuerpo. El borde anterior del pie presenta a cada lado unos pequeños palpos superpuestos de color blanco opaco. Por la transparencia de la concha, se observan también en el interior del animal manchas redondeadas de tamaños variados y de color castaño oscuro, contrastando mucho con el color general del animal.

\section{Etimología}

Volvarina latortuga, toponímico de la isla La Tortuga, donde se ubica su localidad tipo.

\section{Observaciones}

Por el pequeño tamaño y la forma general de la concha, Volvarina latortuga, especie nueva, parece guardar cierta relación con Volvarina banesensis Espinosa \& Ortea, 1999 (7 mm de largo), de la boca del río Banes, costa norte de La Habana, Cuba, de la cual se diferencia porque tiene la espira ligeramente más extendida y aguda, sus pliegues columelares son diferentes en desarrollo y disposición y posee además un patrón de color de la concha distinto. Adicionalmente, V. latortuga, especie nueva, y $V$. ivic, especie nueva, (presente trabajo), se diferencian fácilmente por el desarrollo y disposición de sus pliegues columelares y en el patrón de color de la concha y de los animales vivos.

Con la adición de estas dos nuevas especies, el número de especies válidas del género Volvarina reportadas para Venezuela asciende a 10.

\section{Agradecimientos}

A la Comisión Presidencial de las zonas de utilidad pública y de interés turístico, Dependencias Federales
Isla La Tortuga, Islas Las Tortuguillas, Cayo Herradura y los Palanquines, por el apoyo y la decisión en hacer de la isla un ejemplo de aplicación del conocimiento para el desarrollo racional y sostenible. A Samuel Narciso (FUDENA) y Luz Esther Sánchez (IVIC) por el apoyo logístico en los trabajos de campo y a Augusto Ruiz (IVIC) quien capturó el ejemplar tipo de V. latortuga, especie nueva.

\section{Literatura citada}

Boyer F. 2001. Two Volvarina (Marginellidae) from deep water off northern Honduras. Novapex 2: 3-8.

Boyer F. 2006. Provincia caraibica - Atlantico occidentale. En: Cossignani T (ed). Marginellidae and Cystiscidae of the world, pp. 154-215. L’Informatore Piceno, Ancona.

Cossignani T. 2006. Marginellidae and Cystiscidae of the world, 408 pp. L’Informatore Piceno, Ancona.

Díaz J, J Espinosa \& J Ortea. 1996. Una nueva especie del género Volvarina (Mollusca: Neogastropoda) del mar Caribe colombiano. Avicennia 4/5: 111-114.

Espinosa J \& J Ortea. 1997. Tres nuevas especies del género Volvarina Hinds, 1844 (Mollusca: Neogastropoda: Marginellidae) de las costas de Cuba. Avicennia 6/7: 111116.

Espinosa J \& J Ortea. 1998. Nuevas especies de la familia Marginellidae (Mollusca: Neogastropoda) de Cuba y los cayos de Florida. Avicennia 8-9: 117-134.

Espinosa J \& J Ortea. 1999. Descripción de nuevas marginelas (Mollusca: Neogastropoda: Marginellidae) de Cuba y del Caribe de Costa Rica y Panamá. Avicennia 10/11: 165-176.

Espinosa J \& J Ortea. 2000. Descripción de un género y once especies nuevas de Cystiscidae y Marginellidae (Mollusca: Neogastropoda) del Caribe de Costa Rica. Avicennia 12/ 13: 95-114.

Espinosa J \& J Ortea. 2002. Nuevas especies de margineliformes de Cuba, Bahamas y el mar Caribe de Costa Rica. Avicennia 15: 101-128.

Espinosa J \& J Ortea. 2003. Nuevas especies de moluscos marinos (Mollusca: Gastropoda) del Parque Nacional Guanahacabibes, Pinar del Río, Cuba. Avicennia 16: 143156.

Espinosa J \& J Ortea. 2005. Nuevas especies del género Volvarina Hinds, 1844. En: Espinosa J, J Ortea, M Caballer \& L Moro (eds). Moluscos marinos de la península de Guanahacabibes, Pinar del Río, Cuba, con la descripción de nuevos taxones, Avicennia 18: 45-51.

Espinosa J, J Ortea, R Fernández-Garcés \& L Moro. 2007. Adiciones a la fauna de moluscos marinos de la península de Guanahacabibes (I), con la descripción de nuevas especies. Avicennia 19: 63-88.

Gracia A \& F Boyer. 2004. Distribution of Volvarina hennequini Boyer, 2001 in the Western Caribbean Sea and description of a new bathyal Volvarina from northern 
Colombia. Novapex 5: 79-84.

Lipe B. 1991. Marginellas, 40 pp. The Shell Store, St. Petersburg Beach.

Macsotay O \& R Campos. 2001. Moluscos representativos de la plataforma de Margarita, Venezuela, 280 pp. Rivolta, Valencia.

Narciso S, A Prieto \& V Acosta. 2002. Microgasterópodos asociados con el banco natural de la pepitona Arca zebra (Swaisson, 1833: Mollusca: Bivalvia) ubicado en la localidad de Chacopata, Estado Sucre, Venezuela. Ciencias Marinas 31(1A): 119-124.

Ortea J \& J Espinosa. 1998. Dos nuevas especies de moluscos marinos (Mollusca: Gastropoda) recolectadas en los subarchipiélagos Jardines del Rey y Jardines de la Reina, descritas en honor de los Reyes de España por su primera visita a Cuba. Avicennia 8/9: 1-6.

Princz D. 1978. Moluscos gasterópodos y pelecípodos del Edo. Nueva Esparta, Venezuela. Memorias de la Sociedad de Ciencias Naturales de La Salle 50: 171-222.
Ramos H \& G Robaina. 1994. Contribución al conocimiento de los moluscos gasterópodos y pelecípodos de la bahía de Mochima, Estado Sucre, Venezuela. Memorias de la Sociedad de Ciencias Naturales de La Salle 54 (141): 95106.

Rosenberg G. 2009. Malacolog 4.1.1: A Database of Western Atlantic Marine Mollusca. [WWW database (version 4.1.1)]. [en línea] <http://www.malacolog.org>

Talavera F \& D Princz. 1985. Marginella lasallei spec. nov. y revisión de la familia Marginellidae en el mar venezolano. Bolletino Malacologico 20: 273-282.

Wakefield A, F Boyer \& T McCleery. 2002. Review of the genus Pachybathron Gaskoin, 1853 (Gastropoda: Cystiscidae). Novapex 3: 65-81.

Weisbord NE. 1962. Late Cenozoic gastropods from northern Venezuela. Bulletins of American Paleontology 42(193): 1-672.

Recibido el 7 de abril de 2009 y aceptado el 12 de junio de 2009 